\title{
Efficient and Secure Authentication Scheme Based on Certificateless Aggregate Signature in Space Networks
}

\author{
Hu Zhiyan ${ }^{1+}$, Du Xuehui ${ }^{1}$ and Cao Lifeng ${ }^{1}$ \\ ${ }^{1}$ State Key Laboratory of Mathematical Engineering and Advanced Computing, Zhengzhou 450001, China
}

\begin{abstract}
According to the requirements of the space networks for computing cost, communication cost and security, a new efficient certificateless aggregate signature algorithm is proposed. The signature length is only 2 group elements, the verification stage requires only 3 pairing operations, and the algorithm is proved secure based on the computational Diffie-Hellman problem under the random oracle model. Compared with the other similar algorithm, this algorithm achieves higher security with higher efficiency and lower cost of communication. Then according to the signature algorithm proposed in this paper, an authentication scheme with the function of mutual authentication, aggregate authentication and group key agreement is presented. The analysis results show that the scheme is secure and efficient, especially suitable for the large-scale space networks.
\end{abstract}

Keywords: space networks, certificateless cryptography, aggregate signature, authentication

\section{Introduction}

The space network structures with double plane of heaven and earth, which is based on the ground network and expanding with space-based network. The network consists of space-based backbone network, space-based access network and ground-based node network. As a result of its complex structure, it has the characteristics of heterogeneous, intermittent connectivity, large scale and high exposure, which brings great challenges to access authentication for space networks. It is required that not only to achieve rapid and safety verification, but also to ensure low communication cost to suit the unique characteristic of space networks.

The use of traditional public key cryptosystems (PKC) [1-6] to realize authentication for the space network, which brings the problem of complex certificate management and produces a large amount of computational and storage overhead. As a result of it uses the public key infrastructure (PKI) as a trusted third party to distribute digital certificates, it is not suitable for deployment in resource constrained space networks. Since Shamir proposed the identity-based public key cryptography (ID-PKC) [7], a large number of identity-based authentication scheme [8-11] has been proposed to simplify the certificate management pressure for PKI which brings the key escrow problem, it cannot satisfy the demand of high security in space networks.

In order to overcome the shortcoming of key escrow problem in the ID-PKC and complex certificate management in the PKC, Al-Riyami and Paterson[12] proposed certificateless public key cryptosystem (CLPKC). The same year, Boneh et al. [13] first proposed the concept of aggregate signature in EUROCRYPT, it can aggregate multiple signatures to form a signature, so that only need to verify the aggregate signature to achieve verification. Gong et al. [14] first proposed two certificateless aggregate signature (CLAS) scheme combining aggregate signature algorithm with CL-PKC to achieve the purpose of verification, but bilinear pairing is proportional to the number of signers, there exists a problem of big calculation. Zhang et al. [15] proposed the scheme which pairings operation is reduced, but the length of the signature and the number of

\footnotetext{
+ Corresponding author. Tel.: +8615617764010; fax: +0371-81630094.
}

E-mail address: huzhiyan1234@126.com 
signature is linear correlated, the communication cost is high. The scheme of Zhang et al. [16] used 5 pairing operations to realize aggregate authentication, scheme of Liu [17] is more efficient which only needs 3 pairing operations but introduces state information. Although the scheme of Xiong et al. [18] requires only 3 pairing operation, the signature length is long and the security is poor. Although the scheme of Chen et al. [19] achieves high security, but there exists a shortcoming of introduction of state information. The scheme proposed by $\mathrm{He}$ et al. [20] overcomes the defects of state information, but the computational overhead is relatively large. And the length of the [18-20] aggregate signature is $n+1$ group elements, the communication cost is larger. In summary, the existing authentication scheme based on certificateless aggregation signature scheme in the communication overhead, computation cost and security cannot adapt to the characteristics of the space network that needs to ensure the safety at the same time to achieve fast and efficient authentication.

We first introduce the background knowledge of CLAS scheme, and then propose a new and efficient CLAS algorithm, which proves to be safe based on the $\mathrm{CDH}$ problem in the random oracle model. Compared with the existing schemes, this scheme reduces the computational cost of verification and improves the communication efficiency while ensures the security. Finally, based on the CLAS scheme proposed in this paper, we propose an efficient and secure authentication scheme which is suitable for concurrent access authentication for a number of users in the space network.

\section{Preliminaries}

\subsection{Bilinear Maps}

Let $l$ be a security parameter, $q$ is a prime number of $l-b i t, G_{1}$ represents a cyclic additive group of order $q, G_{2}$ represents a cyclic additive group of order $q, G_{T}$ represents a cyclic multiplicative group of the same order, $P$ is a generator of $G_{1}, Q$ is a generator of $G_{2}$, we call map $e: G_{1} \times G_{2} \rightarrow G_{T}$ a bilinear map if the following properties are satisfied:

(1) Bilinearity: for all $a, b \in Z_{q}^{*}$, such that $e(a P, b Q)=e(P, Q)^{a b}$;

(2) Non-degeneracy: exist $P \in G_{1}, Q \in G_{2}$, such that $e(P, Q) \neq 1$;

(3) Computability: $\forall P, Q \in G_{1}$, it's efficient to compute $e(P, Q)$.

\subsection{Mathematical difficulties}

Computational Diffie-Hellman problem $(\mathrm{CDH}): G_{1}$ is a cyclic additive group of order $q$ with the generator $P$, the CDH problem is to compute $g^{a b}$ when $\left(\mathrm{g}^{a}, \mathrm{~g}^{b}\right)$ is given, $a, b \in Z_{q}^{*}$.

\section{System model}

In space networks, the system model is composed of space-based access network, space-based backbone networks, ground-based node network and base station (BS). As shown in Figure 1. The base station completes the work of system initialization. Ground nodes makes a request to the space-based network nodes when it's in the space-based network node's coverage range. Then ground nodes and space-based network nodes can communicate safely with each other after mutual authentication and key establishment.

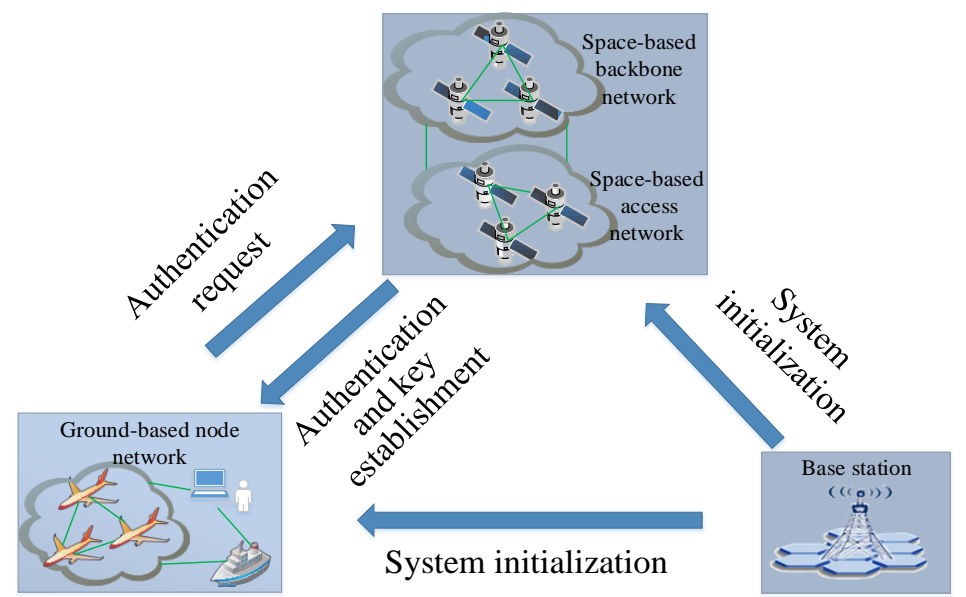

Figure. 1: Simplified model of space network authentication system 


\section{An efficient CLAS algorithm}

We propose an efficient CLAS algorithm which consists of 7 polynomial time algorithms:

Setup : KGC uses the security parameters $k$ to generate system parameter sets and the system master key as follows:

(1) Generate a cyclic additive group $G_{1}$ of $\operatorname{order} q$, a cyclic multiplicative group $G_{2}$ of the same order and a bilinear map $e: G_{1} \times G_{1} \rightarrow G_{2}$.

(2) KGC selects $\lambda \in Z_{q}^{*}$ as the system master key and selects two random generator $P, Q \in G_{1}$. Then computes $P_{p u b}=\lambda P$.

(3) KGC sets three secure hash functions $H_{0}:\{0,1\}^{*} \rightarrow G_{1}, H_{1}, H_{2}:\{0,1\}^{*} \rightarrow Z_{q}^{*}$.

(4) KGC publishes paras $=\left(G_{1}, G_{2}, e, P, Q, P_{p u b}, H_{0}, H_{1}\right)$ and keeps master key $\lambda$ secret.

Partial-private-key generation $G e n_{P P K}$ : It is operated by KGC with a user's identity $I D_{i}$, system parameters paras and master key $\lambda$ to generate user's partial private key $D_{\mathrm{i}}$. Computes $Q_{i}=H_{0}\left(I D_{i}\right)$ and outputs $D_{i}=\lambda Q_{i}$.

Key generation $G e n_{K}$ : It is operated by a user with the user's identity $I D_{i}$ and a random number $x_{i} \in Z_{q}^{*}$ to generate the whole private key $\left(x_{i}, D_{i}\right)$ and public key $P_{i}=x_{i} P$.

Sign: It is operated by each user with their own identity $I D_{i}$, message $M_{i} \in \mu$, plaintext space $\mu=\{0,1\}^{*}$, public key $P_{i}$ and private key $\left(x_{i}, D_{i}\right)$ to generate a valid signature $\sigma_{i}$ by following steps:

(1) Choose $r_{i} \in Z_{q}^{*}$ and compute $R_{i}=r_{i} P$.

(2) Compute $h i=H_{2}\left(M_{i}\left\|I D_{i}\right\| P_{i} \| R_{i}\right), t_{i}=H_{1}\left(M_{i}\left\|I D_{i}\right\| P_{i} \| R_{i}\right)$.

(3) Compute $S_{i}=D_{i}+x_{i} t_{i} Q+\left(h_{i} x_{i}+r_{i}\right) P_{p u b}$.

(4) Output signature $\sigma_{i}=\left(R_{i}, S_{i}\right)$.

Single verification SingleVerify: Given the user's identity $I D_{i}$, the corresponding public key $P_{i}$ and the aggregate signature $\sigma_{i}$ on message $M_{i}$. The verifier computes $h i=H_{2}\left(M_{i}\left\|I D_{i}\right\| P_{i} \| R_{i}\right), t_{i}=H_{1}\left(M_{i}\left\|I D_{i}\right\| P_{i} \| R_{i}\right)$, $Q_{i}=H_{0}\left(I D_{i}\right)$.Check equation $e\left(P, S_{i}\right)=e\left(P_{p u b}, Q_{i}+h_{i} P_{i}+R_{i}\right) e\left(Q, P_{i} t_{i}\right)$, if it holds, output true, otherwise false.

Aggregate: Aggregate signature generator (any user can serve as an aggregate signature generator) runs the algorithm. On input a set of $\mathrm{n}$ users with identity $\operatorname{set}\left\{I D_{1}, I D_{2}, \ldots, I D_{\mathrm{n}}\right\}$, the corresponding public key set $\left\{\mathrm{P}_{1}, \mathrm{P}_{2}, \ldots, \mathrm{P}_{\mathrm{n}}\right\}$ and $\mathrm{n}$ distrinct message-signature pairs $\operatorname{set}\left\{\left(M_{1}, \sigma_{1}\right),\left(M_{2}, \sigma_{2}\right), \ldots,\left(M_{n}, \sigma_{n}\right)\right\}$, the generator computes $R=\sum_{i=1}^{n} R_{i}, S=\sum_{i=1}^{n} S_{i}$ and outputs the aggregate signature $\sigma=(R, S)$ on message set $\left\{M_{1}, M_{2}, \ldots \ldots, M_{n}\right\}$.

AggregateVerify: Given $\mathrm{n}$ users with identity $\operatorname{set}\left\{I D_{1}, I D_{2}, \ldots, I D_{\mathrm{n}}\right\}$, the corresponding public key set $\left\{\mathrm{P}_{1}, \mathrm{P}_{2}, \ldots, \mathrm{P}_{\mathrm{n}}\right\}$ and the aggregate signature $\sigma=(R, S)$, the verifier performs the following steps:

(1) For all $i(1 \leq i \leq n)$, computes $h_{i}=H_{2}\left(M_{i}\left\|I D_{i}\right\| P_{i} \| R_{i}\right), \quad t_{i}=H_{1}\left(M_{i}\left\|I D_{i}\right\| P_{i} \| R_{i}\right), Q_{i}=H_{0}\left(I D_{i}\right)$.

(2) Check $e(P, S)=e\left(P_{p u b}, \sum_{i=1}^{n}\left(Q_{i}+h_{i} P_{i}\right)+R\right) e\left(Q, \sum_{i=1}^{n} P_{i} \cdot t_{i}\right)$.If holds, output true, otherwise false.

\section{Security analysis}

\subsection{Correctness analysis}

$$
\begin{aligned}
& e(P, S)=e\left(P, \sum_{i=1}^{n}\left(D_{i}+x_{i} t_{i} Q+\left(h_{i} x_{i}+r_{i}\right) P_{p u b}\right)\right) \\
& =e\left(P, \sum_{i=1}^{n}\left(D_{i}+\left(h_{i} x_{i}+r_{i}\right) P_{p u b}\right)\right) e\left(P, \sum_{i=1}^{n} x_{i} t_{i} Q\right) \\
& =e\left(P_{p u b}, \sum_{i=1}^{n} Q_{i}+\left(h_{i} x_{i}+r_{i}\right) P\right) e\left(Q, \sum_{i=1}^{n} P_{i} \cdot t_{i}\right) \\
& =e\left(P_{p u b}, \sum_{i=1}^{n}\left(Q_{i}+h_{i} P_{i}\right)+R\right) e\left(Q, \sum_{i=1}^{n} P_{i} \cdot t_{i}\right)
\end{aligned}
$$

\subsection{Security proof}

Theorem 1. In the random oracle model, if a polynomial time type I adversary $A_{1}$ who has the nonnegligible advantage $\varepsilon$ forging the proposed CLAS scheme with at most $q_{H_{i}}$ times $H_{i}\left(H_{i}=H_{0}, H_{1}, H_{2}\right)$ queries, $q_{k}$ times $P P K\left(I D_{i}\right)$ queries, $q_{p}$ times $P K\left(I D_{i}\right)$ queries, $q_{s}$ times $S\left(M_{i}, I D_{i}, P_{i}\right)$ queries, there is an algorithm can solve $\mathrm{CDH}$ problem in time $t^{\prime}=t+\left(q_{H_{0}}+q_{k}+q_{p}+4 q_{s}\right) t_{s m}$ and probability $\varepsilon^{\prime}=\frac{1}{q_{k}+n}\left(1-\frac{1}{q_{k}+n}\right)^{q_{k}+n-1}$, where 
$n$ is the number of signer, $t_{s m}$ is the time spent on scalar multiplication in group $G_{1}$.

Proof: It is assumed that a type I adversary can break the proposed CLAS with non-negligible advantage. Let $C$ be an algorithm that can solve $\mathrm{CDH}$ problem. Given a $\mathrm{CDH}$ problem instance $(P, a P, b P)$, $C$ can solve $\mathrm{CDH}$ problem (compute $a b P$ ) by interacting with $A_{1}$

Setup :The algorithm $C$ sets $P_{p u b}=a P$, chooses $w \in Z_{q}^{*}$ and sets $Q=w P$, returns set paras $=\left(G_{1}, G_{2}, e, P, Q, P_{p u b}, H_{0}, H_{1}, H_{2}\right)$ to $A_{1}$.

Query : Hash functions $H_{0}, H_{1}, H_{2}$ are random oracles. It is assumed that $A_{1}$ can make at most $q_{H_{i}}$ times $H_{i}\left(H_{i}=H_{0}, H_{1}, H_{2}\right)$ queries, $q_{k}$ times $P P K\left(I D_{i}\right)$ queries, $q_{p}$ times $P K\left(I D_{i}\right)$ queries and $q_{s}$ times $S\left(M_{i}, I D_{i}, P_{i}\right)$ queries. $A_{1}$ can make following queries in an adaptive way.

$H_{0}$ queries: $C$ manages an initially empty list $L_{0}$. The format of $L_{0}$ is $\left(I D_{i}, \alpha_{i}, Q_{i}, c_{i}\right)$. When $C$ receiving the $H_{0}$ query on $I D_{i}, C$ returns the corresponding value to $A_{1}$ if the corresponding entry already exists in the list $L_{0}$.Otherwise, $C$ tosses a coin $c_{i} \in\{0,1\} \quad\left(\operatorname{Pr}\left[c_{i}=0\right]=\delta, \operatorname{Pr}\left[c_{i}=1\right]=1-\delta\right)$. When $c_{i}=0, C$ randomly picks $\alpha_{i} \in Z_{q}^{*}$, sets $Q_{i}=\alpha_{i} b P$ and returns $Q_{i}$ to adversary $A_{1}$, then adds $\left(I D_{i}, \alpha_{i}, Q_{i}, c_{i}\right)$ to $L_{0}$. When $c_{i}=1$, $C$ randomly picks $\alpha_{i} \in Z_{q}^{*}$, sets $Q_{i}=\alpha_{i} P$ and returns $Q_{i}$ to adversary $A_{1}$, then adds (IDi, $\left.\alpha_{i}, Q_{i}, c_{i}\right)$ to $L_{0}$.

$H_{1}$ queries: $C$ manages an initially empty list $L_{1}$. The format of $L_{1}$ is $\left(M_{i}, I D_{i}, P_{i}, R_{i}, t_{i}\right)$. When $C$ receiving the $H_{1}$ query on $t_{i}, C$ returns the corresponding value to $A_{1}$ if the corresponding entry already exists in the list $L_{1}$. Otherwise, $C$ randomly picks $t_{i} \in Z_{q}^{*}$, returns $t_{i}$ to adversary $A_{1}$ and adds $\left(M_{i}, I D_{i}, P_{i}, R_{i}, t_{i}\right)$ to $L_{1}$.

$H_{2}$ queries: $C$ manages an initially empty list $L_{2}$. The format of $L_{2}$ is $\left(M_{i}, I D_{i}, P_{i}, R_{i}, h_{i}\right)$. When $C$ receiving the $H_{2}$ query on $h_{i}, C$ returns the corresponding value to $A_{1}$ if the corresponding entry already exists in the list $L_{2}$. Otherwise, $C$ randomly picks $h_{i} \in Z_{q}^{*}$, returns $h_{i}$ to adversary $A_{1}$ and adds $\left(M_{i}, I D_{i}, P_{i}, R_{i}, h_{i}\right)$ to $L_{2}$.

Partial private key $P P K\left(I D_{i}\right)$ queries: $C$ manages an initially empty list $L k$. The format of $L_{k}$ is $\left(I D_{i}, x_{i}, P_{i}, D_{i}\right)$.When $C$ receiving the $P P K\left(I D_{i}\right)$ query, $C$ returns the corresponding value to $A_{1}$ if the corresponding entry already exists in the list $L_{k}$. Otherwise, $C$ makes $H_{0}$ queries on $I D_{i}$ to obtain the corresponding $\left(I D_{i}, \alpha_{i}, Q_{i}, C_{i}\right)$ at first, then performs the following steps:

(1) If $c_{i}=0, C$ aborts.

(2) If there is a four tuples $\left(I D_{i}, x_{i}, P_{i}, D_{i}\right)$ in the list $L_{k}, C$ sets $D_{i}=\alpha_{i} P_{p u b}$ and returns $D_{i}$.

(3) Otherwise, $C$ computes $D_{i}=\alpha_{i} P_{p u b}$, sets $x_{i}=P_{i}=\perp$, returns the answer $D_{i}$ and adds $\left(I D_{i}, x_{i}, P_{i}, D_{i}\right)$ to the list $L_{k}$.

Public key $P K\left(I D_{i}\right)$ queries: When $C$ receiving the $P K\left(I D_{i}\right)$ query, $C$ returns the corresponding value to $A_{1}$ if the query has been made before, otherwise, $C$ performs the following steps:

(1) If there is a four tuples $\left(I D_{i}, x_{i}, P_{i}, D_{i}\right)$ in the list $L_{k}$, the public key is $\perp, C$ randomly picks $x_{i}^{\prime} \in Z_{q}^{*}$, sets $P_{i}^{\prime}=x_{i}^{\prime} P$ and updates tuples $\left(I D_{i}, x_{i}, P_{i}, D_{i}\right)$ to $\left(I D_{i}, x_{i}^{\prime}, P_{i}^{\prime}, D_{i}\right)$.

(2) Otherwise, $C$ randomly chooses $x_{i} \in Z_{q}^{*}$, sets $P_{i}=x_{i} P$, returns $P_{i}$ as the answer, sets $D_{i}=\perp$ and adds the tuples $\left(I D_{i}, x_{i}, P_{i}, D_{i}\right)$ to the list $L_{k}$.

Secret value $S V\left(I D_{i}\right)$ queries: When $C$ receiving the $S V\left(I D_{i}\right)$ query, $C$ first checks whether there is a corresponding $x_{i}$ in the list $L_{k}$, if exists, $C$ returns $x_{i}$ to $A_{1}$. Otherwise, $C$ makes $P K\left(I D_{i}\right)$ queries to find the corresponding $x_{i}$ and returns it to $A_{1}$.

Public key substitution $\operatorname{PKR}\left(I D_{i}, P_{\mathrm{i}}^{\prime}\right)$ queries: When $C$ receiving the $P K R\left(I D_{i}, P_{\mathrm{i}}^{\prime}\right)$ query, $C$ first checks whether there is a corresponding tuple $\left(I D_{i}, x_{i}, P_{i}, D_{i}\right)$ in the list $L_{k}$, if exists and $P_{i} \neq \perp, C$ updates $P_{i}$ in the tuple to $P_{i}^{\prime}$. Otherwise, $C$ makes $P K\left(I D_{i}\right)$ queries to find $P_{i}$ and updates $P_{i}$ to $P_{i}^{\prime}$.

Sign $S\left(M_{i}, I D_{i}, P_{i}\right)$ queries: When $C$ receiving the $S\left(M_{i}, I D_{i}, P_{i}\right)$ query, $C$ makes $H_{0}, H_{1}$ and $H_{2}$ queries to obtain $\left(I D_{i}, \alpha_{i}, Q_{i}, c_{i}\right)$ from the list $L_{0},\left(M_{i}, I D_{i}, P_{i}, R_{i}, t_{i}\right)$ from the list $L_{1}$ and $\left(M_{i}, I D_{i}, P_{i}, R_{i}, h_{i}\right)$ from the list $L_{2}$. Then forms the signature.

(1) If $c_{i}=0, C$ randomly chooses $r_{i} \in Z_{q}^{*}$, sets $R_{i}=r_{i} P-\alpha_{i} b P$, computes $S_{i}=x_{i t} Q+\left(h_{i} x_{i}+r_{i}\right) P_{p u b}$ and outputs the signature $\sigma_{i}=\left(R_{i}, S_{i}\right)$.

(2) If $c_{i}=1, C$ randomly chooses $r_{i} \in Z_{q}^{*}$, sets $R_{i}=r_{i} P$, computes $S_{i}=\alpha_{i} P_{p u b}+x_{i} t_{i} Q+\left(h_{i} x_{i}+r_{i}\right) P_{p u b}$ and outputs signature $\sigma_{i}=\left(R_{i}, S_{i}\right)$. 
Forgery: $A_{1}$ outputs the forged signature $\sigma^{*}=\left(R^{*}, S^{*}\right)$ on the users set $U^{*}=\left\{U_{1}^{*}, U_{2}^{*}, \ldots U_{\mathrm{n}}^{*}\right\}$, the identity set $L_{I D}^{*}=\left\{I D_{1}^{*}, I D_{2}^{*}, \ldots, I D_{\mathrm{n}}^{*}\right\}$, the corresponding public key set $L_{P K}^{*}=\left\{P_{1}^{*}, P_{2}^{*}, \ldots, P_{\mathrm{n}}^{*}\right\}$ and the message set $L_{M}^{*}=\left\{M_{1}^{*}, M_{2}^{*}, \ldots, M_{\mathrm{n}}^{*}\right\}$. And $C$ obtains $\left(I D_{i}, \alpha_{i}, Q_{i}, C_{i}\right)$ from the list $L_{0},\left(M_{i}, I D_{i}, P_{i}, R_{i}, t_{i}\right)$ from the list $L_{1}$, $\left(M_{i}, I D_{i}, P_{i}, R_{i}, h_{i}\right)$ from the list $L_{2}$. It is required that there is at least one $i \in[1, n]$ that $A_{1}$ has not made $P P K\left(I D_{i}\right)$ queries $\left(c_{i}^{*}=0\right)$ and $S\left(M_{i}, I D_{i}, P_{i}\right)$ queries. Without losing generality, we assume that $i=1$. The forged signature $\sigma^{*}=\left(R^{*}, S^{*}\right)$ satisfies $e(P, S)=e\left(P_{p u b}, \sum_{i=1}^{n}\left(Q_{i}+h_{i} P_{i}\right)+R\right) e\left(Q, \sum_{i=1}^{n} P_{i} \cdot t_{i}\right)$. So we can get $e\left(P, S^{*}\right)=e\left(P_{p u b}, \sum_{i=1}^{n}\left(Q_{i}^{*}+h_{i}^{*} P_{i}^{*}\right)+R^{*}\right) e\left(Q, \sum_{i=1}^{n} P_{i}^{*} \cdot t_{i}^{*}\right)$

$=e\left(P, \alpha_{1}^{*} a b P+h_{1}^{*} x_{1}^{*} P_{p u b}+r_{1}^{*} P_{p u b}\right) e\left(P, \sum_{i=2}^{n}\left(\alpha_{i}^{*} P_{p u b}+h_{i}^{*} x_{i}^{*} P_{p u b}+r_{i}^{*} P_{p u b}\right)\right) e\left(P, Q \sum_{i=1}^{n} x_{i}^{*} \cdot t_{i}^{*}\right)$

and then compute $a b P=\left(S^{*}-h_{1}^{*} x_{1}^{*} P_{p u b}-r_{1}^{*} P_{p u b}-\sum_{i=2}^{n}\left(\alpha_{i}^{*} P_{p u b}+h_{i}^{*} x_{i}^{*} P_{p u b}+r_{i}^{*} P_{p u b}\right)-Q \sum_{i=1}^{n} x_{i}^{*} \cdot t_{i}^{*}\right) \alpha_{1}^{*-1}$. Otherwise, $C$ aborts.

$C$ can compute $a b P$ through the following events:

(1) $E 1: C$ does not abort when $A_{1}$ makes $P P K\left(I D_{i}\right)$ queries.

(2) $E 2: A_{1}$ produces a valid aggregate signature.

(3) E3: When $E 2$ happens, $c_{1}^{*}=0$ and $c_{i}^{*}=1$ for $i \in[2, n]$ are required.

The success probability of $C$ is $\operatorname{Pr}[E 1 \wedge E 2 \wedge E 3]=\operatorname{Pr}[E 1] \operatorname{Pr}[E 2 \mid E 1] \operatorname{Pr}[E 3 \mid E 1 \wedge E 2]$.

$C$ does not abort with a probability of $1-\delta$ during partial private key queries. $\operatorname{Pr}[E 1] \geq(1-\delta)^{q_{k}}$ as a result of $A_{1}$ makes $q_{k}$ times partial private key queries. $C$ does not abort when making $P P K\left(I D_{i}\right)$ queries, it is difficult for adversary $A_{1}$ to distinguish between simulated and real environments, so $\operatorname{Pr}[E 2 \mid E 1]=\varepsilon \cdot \operatorname{Pr}[E 3 \mid E 1 \wedge E 2]=\delta(1-\delta)^{n-1}$ when $c_{1}^{*}=0$ and $c_{i}^{*}=1$ for all $i \in[2, n]$. In the end, we can get $\quad \varepsilon^{\prime}=\operatorname{Pr}[E 1 \wedge E 2 \wedge E 3] \geq \delta(1-\delta)^{q_{k}+n-1} \varepsilon \quad, \quad$ when $\quad \varepsilon=\frac{1}{q_{k}+n} \quad, \quad$ the maximum value of $\varepsilon^{\prime}$ is $\varepsilon_{\max }^{\prime}=\frac{1}{q_{k}+n}\left(1-\frac{1}{q_{k}+n}\right)^{q_{k}+n-1}$.

The operation time of algorithm $C$ is the sum of forging time of adversary $A_{1}$, query time of $q_{H_{i}}$ times $H_{i}\left(H_{i}=H_{0}, H_{1}, H_{2}\right)$ queries, the time of $q_{k}$ times $P P K\left(I D_{i}\right)$ queries, the time of $q_{p}$ times $P K\left(I D_{i}\right)$ queries, the time of $q_{s}$ times $S\left(M_{i}, I D_{i}, P_{i}\right)$ queries. Every $q_{H_{0}}$ queries, $q_{k}$ queries or $q_{p}$ queries requires one time scalar multiplication, every $q_{s}$ queries requires 4 times scalar multiplication. So $t^{\prime}=t+\left(q_{H_{0}}+q_{k}+q_{p}+4 q_{s}\right) t_{s m}$.

Therefore if there is a type I adversary who can break the proposed CLAS with non-negligible advantage, $C$ can solve $\mathrm{CDH}$ problem.

Theorem 2. In the random oracle model, if a polynomial time type II adversary $A_{2}$ who has the nonnegligible advantage $\varepsilon$ forging the proposed CLAS scheme with at most $q_{H i}$ times $H_{i}\left(H_{i}=H_{0}, H_{1}, H_{2}\right)$ queries, $q_{v}$ times $S V\left(I D_{i}\right)$ queries, $q_{p}$ times $P K\left(I D_{i}\right)$ queries, $q_{s}$ times $S\left(M_{i}, I D_{i}, P_{i}\right)$ queries, there is an algorithm can solve $\mathrm{CDH}$ problem in time $t^{\prime}=t+\left(q_{v}+q_{p}+4 q_{s}\right) t_{s m}$ and probability $\varepsilon^{\prime}=\frac{1}{q_{k}+n}\left(1-\frac{1}{q_{k}+n}\right)^{q_{k}+n-1}$.

Proof: Be similar to the theorem 1.

\section{The authentication scheme based on CLAS}

In this paper, we design an authentication scheme based on CLAS scheme, which includes the system initialization phase, access authentication in the space network and the aggregate authentication phase.

\subsection{System initialization}

The base station complete the system initialization phase, which consists of setup, partial-private-key generation, key generation in section 4. So I won't go into much detail here. All network nodes (including network access node, etc.) complete the system initialization before deployment.

\subsection{Access authentication in the space network}

\subsubsection{Authentication process}

Access authentication will happen between ground nodes and space-based access nodes, space-based access nodes and space-based backbone nodes, ground nodes and space-based backbone nodes. The 
authentication process is similar. It is assumed that the access authentication and key agreement process is completed by the user terminal UN (include ground nodes) and the network access node AN (include spacebased access nodes and space-based backbone nodes) after the system initialization phase is finished. Before the formal process of the access authentication, it is necessary to select the access node. When the node UN is within the coverage of AN, it can receive the periodic broadcast message of AN, the format of Broadcastmessage is $A N \rightarrow^{*}: I D_{A N}$, paras $_{A N}, \sigma_{A N}\left(I D_{A N}\right.$, paras $\left._{A N}\right)$. The UN can check whether they are in the coverage range of AN When the UN receives this message. The UN can get system parameters paras ${ }_{A N}$ and the identity of AN $I D_{A N}$. Compared with the parameters owned by AN, the UN can check whether the parameters are the same to confirm the identity of AN. The UN may be within the coverage of multiple ANs. When a UN receives a broadcast message transmitted by a plurality of ANs, the AN selects the highest signal strength to issue an access request. Access authentication process requires 2 interactions, as shown in Figure 2.

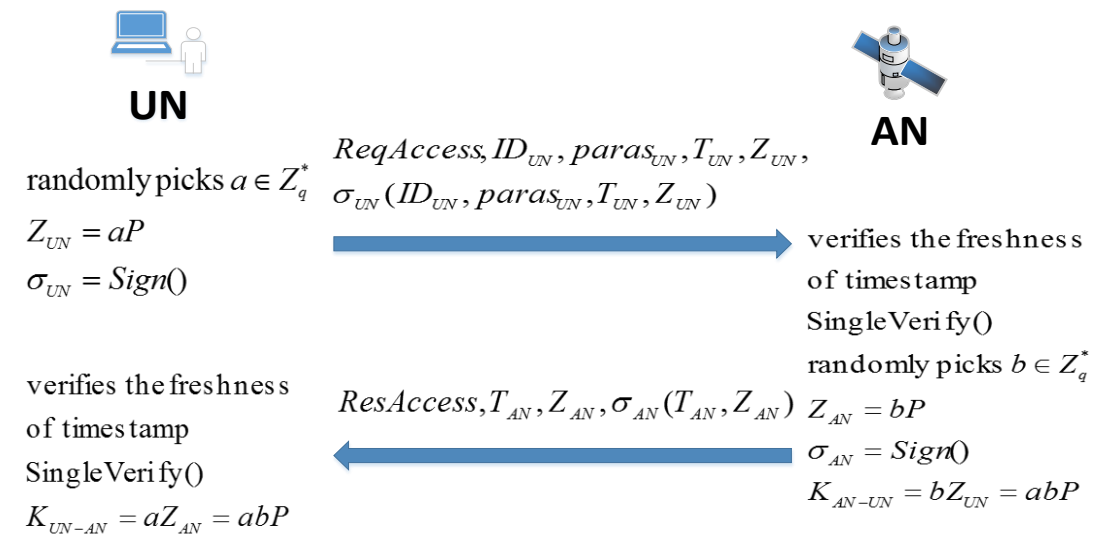

Figure. 2: Access authentication process in space network

(1) The UN randomly chooses $a \in Z_{q}^{*}$, computes $Z_{U N}=a P$, generates the aggregate signature $\sigma_{U N}$ with the proposed CLAS algorithm and send the message $U N \rightarrow$ AN : ReqAccess, $I D_{U N}$, paras $_{U N}, T_{U N}, Z_{U N}, \sigma_{U N}\left(I D_{U N}\right.$, paras $\left._{U N}, T_{U N}, Z_{U N}\right)$ to the AN.

(2) The AN verifies the freshness of timestamp $T_{U N}$ at first when it receives the message. And then it verifies the legality of UN and the message through single verification algorithm SingleVerify(). If it does not pass, the process is abandoned, otherwise, the AN randomly picks $b \in Z_{q}^{*}$, computes $Z_{A N}=b P$ and the signature $\sigma_{A N}$, computes session key $K_{A N-U N}=b Z_{U N}=a b P$ based on the received message. The transmitted message ResAccess is as follows: $A N \rightarrow U N:$ ResAccess, $T_{A N}, Z_{A N}, \sigma_{A N}\left(T_{A N}, Z_{A N}\right)$

(3) The UN verifies the freshness of timestamp at first when it receives the message. And then it verifies the legality of AN through single verification algorithm SingleVerify() to achieve mutual authentication. Computes session key $K_{U N-A N}=a Z_{A N}=a b P$.

\subsubsection{Key update}

In order to enhance the security of the communication between $\mathrm{AN}$ and $\mathrm{UN}$, the session key needs to be updated periodically.

(1) The UN issues key update request ReqRefresh to AN, randomly chooses $a^{\prime} \in Z_{q}^{*}$, computes $Z_{U N}^{\prime}=a^{\prime} P$, signs the message using $\operatorname{Sign}()$, then encrypts the messages with $K_{U N-A N}$ and transmits it to AN. The format of the message is as follows: $U N \rightarrow A N: K_{U N-A N}\left(\right.$ ReqRefresh, $I D_{U N}, T_{U N}, Z_{U N}^{\prime}, \sigma_{U N}\left(\right.$ ReqRefresh, $\left.\left.I D_{U N}, T_{U N}, Z_{U N}^{\prime}\right)\right)$.

(2) The AN decrypts the message with the session key $K_{A N-U N}$, verifies the freshness of timestamp $T_{U N}$ and the validity of the signature. If it passes, the AN randomly picks $b^{\prime} \in Z_{q}^{*}$, computes $Z_{A N}^{\prime}=b^{\prime} P$, signs the message using $\operatorname{Sign}()$ with key refresh response ResRefresh, encrypts the messages with $K_{A N-U N}$ and transmits it to UN. The format of the message is as follows: $A N \rightarrow U N: K_{A N-U N}\left(\right.$ ResRefresh, $T_{A N}, Z_{A N}^{\prime}, \sigma\left(\right.$ ResRefresh $\left.\left., T_{A N}, Z_{A N}^{\prime}\right)\right)$.

The AN and UN can update the session key to $K_{A N-U N}=K_{U N-A N}=a^{\prime} b^{\prime} P$ through one round interaction after mutual authentication. 


\subsection{Aggregate authentication in the space network}

In space networks, there is a situation that the cluster nodes are connected in parallel, such as the battleplane group or the ground cluster user accessing to the satellite. Because the resource of space networks is limited, the overall cost of signature verification affects the overall performance of the authentication service node directly. According to the group key agreement protocol proposed by Sun et al. ${ }^{[21]}$, we design the aggregate authentication scheme to achieve batch verification for cluster nodes and negotiate a group key to realize security communication in group members.

This scheme needs three steps and two rounds to complete authentication and group key agreement:

(1) Every user $U N_{i}(1 \leq i \leq n)$ randomly picks $a_{i} \in Z_{q}^{*}$, computes $L_{i}, Z_{i}$ using other users' public key as follows: $L i=a_{i} x_{0} P, Z i=x_{i}\left(x_{i+1} P-x_{i-1} P\right)$, computes $\sigma_{i}=\left(R i, S_{i}\right)$ based on the proposed CLAS scheme $\operatorname{Sign}() \cdot U_{i}$ sends $\left(\sigma_{i}, L_{i}, Z_{i}\right)$ to AN.

(2) When the AN receives all parameters transmitted from all users $U N_{i}(1 \leq i \leq n)$, it computes parameters that are needed based on Aggregate() and AggragateVerify ().The AN aggregate verifies all users' signatures through $e(P, S)=e\left(P_{p u b}, \sum_{i=1}^{n}\left(Q_{i}+h_{i} P_{i}\right)+R\right) e\left(Q, \sum_{i=1}^{n} P_{i} \cdot t_{i}\right)$. If it passes, the AN randomly picks $a_{0} \in Z_{q}^{*} \quad, \quad$ computes $\quad A_{i}=L_{i} x_{0}^{-1}=a_{i} P, Y=a_{0} P+A_{1}+A_{2}+\ldots+A_{n}, O_{i}=a_{0} A_{i}(1 \leq i \leq n) \quad$ and $Z_{i}^{\prime}=(n-1) Z_{i}+(n-2) Z_{i+1}+\ldots+Z_{i-2}(1 \leq i \leq n)$, computes $\sigma_{0}=\left(R_{0}, S_{0}\right)$ with the algorithm $\operatorname{Sign}()$ and broadcasts $\left(\sigma_{0}, O_{1}, O_{2}, \ldots O n, Y, Z_{1}^{\prime}, Z_{2}^{\prime}, \ldots Z_{n}^{\prime}\right)$ to all users $U N_{i}(1 \leq i \leq n)$ 。

(3) Every user $U N_{i}(1 \leq i \leq n)$ verifies the legality of the AN through the proposed algorithm SingleVerify().

If it passes, each UN computes $K a_{i}=Y-O i a_{i}^{-1}, K b_{i}=n x_{i} x_{i-1} P+Z_{i}^{\prime}$.The group session key is $G K=K a_{i}+K b_{i}=A_{1}+A_{2}+\ldots+A_{n}+x_{i-1} x_{i} P+x_{i} x_{i+1} P+x_{i+1} x_{i+2} P+\ldots+x_{i-2} x_{i-1} P$.

\section{Performance comparison}

At first, we compare the proposed CLAS algorithm in efficiency, communication cost and security with the schemes in literatures [14-20], as shown in Table 1, the efficiency consists of single signature algorithm, aggregate verification. Communication cost includes aggregate signature length, the length of the public key and private key length. Security includes resistance to the first class of adversary and resistance to the second type of adversary. Symbol definitions are shown in table 2. At the same point, all these algorithms used the characteristic of bilinear pairings to realize aggregation verification, and they are based on the certificateless public key cryptosystem to overcome the key escrow problem. At different points, the proposed CLAS scheme in this paper is relatively efficient in the single signature algorithm. The aggregate verification only needs 3 pairing operations which is independent of the number of signers, the efficiency is high. The private key length is one group element length, aggregate signature length is two group element length, and the communication cost is low. What's more, our scheme can resist the first and second types of adversaries in safety. Compared with other schemes, it can achieve low communication cost and high efficiency while ensuring security.

Table 1: Comparison of CLAS schemes

\begin{tabular}{|c|c|c|c|c|c|c|c|}
\hline \multirow[b]{2}{*}{ Scheme } & \multicolumn{2}{|r|}{ Efficiency } & \multicolumn{3}{|c|}{ Communication cost } & \multicolumn{2}{|c|}{ Security } \\
\hline & $\begin{array}{l}\text { Single signature } \\
\text { algorithm }\end{array}$ & Aggregate verification & $\begin{array}{l}\text { aggregate } \\
\text { signature } \\
\text { length }\end{array}$ & $\begin{array}{c}\text { public } \\
\text { key } \\
\text { length }\end{array}$ & $\begin{array}{c}\text { private } \\
\text { key } \\
\text { length }\end{array}$ & $\begin{array}{l}\text { First } \\
\text { type }\end{array}$ & $\begin{array}{l}\text { Second } \\
\text { type }\end{array}$ \\
\hline [14-1] & $2 \mathrm{SM}+1 \mathrm{H}+1 \mathrm{Add}$ & $\mathrm{nH}+(2 \mathrm{n}+1) \mathrm{P}$ & $(\mathrm{n}+1) \mathrm{L}$ & $2 \mathrm{~L}$ & $\mathrm{~L}$ & $\sqrt{ }$ & - \\
\hline [14-1] & $3 \mathrm{SM}+2 \mathrm{H}+2 \mathrm{Add}$ & $2 n H+(n+2) P$ & $2 \mathrm{~L}$ & $2 \mathrm{~L}$ & $2 \mathrm{~L}$ & - & - \\
\hline$[15]$ & $3 \mathrm{SM}+2 \mathrm{H}+2 \mathrm{Add}$ & $(n+3) P+(2 n+1) H+(2 n-2)$ Add & $(n+1) L$ & $\mathrm{~L}$ & $\mathrm{~L}$ & $\sqrt{ }$ & $\sqrt{ }$ \\
\hline [16] & $5 \mathrm{SM}+4 \mathrm{H}+4 \mathrm{Add}$ & $2 n S M+5 P+(3 n+3) H+(4 n-4)$ Add & $2 \mathrm{~L}$ & $\mathrm{~L}$ & $2 \mathrm{~L}$ & $\sqrt{ }$ & $\sqrt{ }$ \\
\hline [17] & $4 \mathrm{SM}+2 \mathrm{H}+2 \mathrm{Add}$ & $2 n S M+3 P+(2 n+1) H+(2 n-2) A d d$ & $2 \mathrm{~L}$ & $\mathrm{~L}$ & $\mathrm{~L}$ & $\sqrt{ }$ & $\sqrt{ }$ \\
\hline$[18]$ & $3 \mathrm{SM}+1 \mathrm{H}+2 \mathrm{Add}$ & $2 n S M+3 P+2 n H+(3 n-2) A d d$ & $(n+1) L$ & $\mathrm{~L}$ & $\mathrm{~L}$ & $\sqrt{ }$ & $x$ \\
\hline [19] & $4 \mathrm{SM}+4 \mathrm{H}+2 \mathrm{Add}$ & $2 n S M+4 P+(3 n+2) H+(3 n-3)$ Add & $(n+1) L$ & $\mathrm{~L}$ & $\mathrm{~L}$ & $\sqrt{ }$ & $\sqrt{ }$ \\
\hline [20] & $3 \mathrm{SM}+2 \mathrm{H}+2 \mathrm{Add}$ & $2 n S M+3 P+3 n H+(3 n-2)$ Add & $(\mathrm{n}+1) \mathrm{L}$ & $\mathrm{L}$ & $\mathrm{L}$ & $\sqrt{ }$ & $\sqrt{ }$ \\
\hline Our scheme & $3 \mathrm{SM}+2 \mathrm{H}+2 \mathrm{Add}$ & $2 n S M+3 P+3 n H+(2 n-1) A d d$ & $2 \mathrm{~L}$ & $\mathrm{~L}$ & $\mathrm{~L}$ & $\sqrt{ }$ & $\sqrt{ }$ \\
\hline
\end{tabular}

Table 2: Symbol definition

\begin{tabular}{cc}
\hline Symbol & Definition \\
\hline SM & Scalar multiplication in $G_{1}$ \\
$\mathrm{H}$ & Hash function operation \\
Add & Add in $G_{1}$ \\
$\mathrm{P}$ & Bilinear pairing operation \\
\hline
\end{tabular}




\begin{tabular}{cc}
\hline e & exponentiation in $G_{2}$ \\
MAC & Message authentication code \\
$\mathrm{L}$ & Element length in $\boldsymbol{G}_{1}$ \\
$\sqrt{ }$ & Be able to resist the attack \\
$\times$ & Unable to resist the attack \\
- & Non formal proof \\
\hline
\end{tabular}

The authentication scheme in this paper is compared with the existing authentication schemes (Our scheme achieves aggregate verification and group key agreement, this part only considers the cost on aggregate verification in comparison with other schemes). As shown in Table 3. Compared with [17], although two schemes used 3 pairing to achieve authentication, the interaction times and computational cost is lower in the independent verification. Compared with the literature [20][22], although our scheme computation cost is high in independent verification, the bilinear pairing operation is reduced to constant magnitude in the aggregate authentication, the computation is significantly lower than before. In the aspect of security, the security of this scheme is based on the proposed CLAS algorithm. Therefore, the scheme is more efficient and more suitable for large scale space networks.

Table 3: The comparison of authentication scheme

\begin{tabular}{ccccc}
\hline \multirow{2}{*}{ Scheme } & \multicolumn{2}{c}{ Independent authentication } & \multicolumn{2}{c}{ Aggregate authentication } \\
\cline { 2 - 5 } & Computation cost & $\begin{array}{c}\text { Communication } \\
\text { cost }\end{array}$ & \multicolumn{1}{c}{ Computation cost } & $\begin{array}{c}\text { Communication } \\
\text { cost }\end{array}$ \\
\hline$[17]$ & $3 \mathrm{P}+8 \mathrm{SM}+5 \mathrm{H}+3 \mathrm{Add}+1 \mathrm{MAC}$ & 3 & $\begin{array}{l}3 \mathrm{P}+(2 \mathrm{n}+4) \mathrm{SM}+(2 \mathrm{n}+3) \mathrm{H}+(4 \mathrm{n}- \\
\mathrm{n}+1\end{array}$ \\
{$[20]$} & $2 \mathrm{P}+1 \mathrm{SM}+2 \mathrm{H}+1 \mathrm{Add}$ & 2 & $2 \mathrm{nP}+1 \mathrm{SM}+\mathrm{nAdd}+(\mathrm{n}+1) \mathrm{H}$ & $\mathrm{n}+1$ \\
{$[22]$} & $2 \mathrm{P}+8 \mathrm{SM}$ & 2 & $(\mathrm{n}+1) \mathrm{P}+(5 \mathrm{n}+1) \mathrm{SM}+\mathrm{e}$ & $\mathrm{n}+1$ \\
Our & $3 \mathrm{P}+7 \mathrm{SM}+5 \mathrm{H}+4 \mathrm{Add}$ & 2 & $3 \mathrm{P}+(2 \mathrm{n}+3) \mathrm{SM}+(3 \mathrm{n}+2) \mathrm{H}+(4 \mathrm{n}-$ & $\mathrm{n}+1$ \\
scheme & & 2 & $1) \mathrm{Add}$ & \\
\hline
\end{tabular}

\section{Conclusions and future work}

According to the characteristics of the space network, we propose an efficient CLAS scheme based on bilinear map, and puts forward to an authentication scheme based on it. Compared with the existing CLAS algorithm, our algorithm realizes higher security with the lower communication cost and the higher efficiency. And the authentication scheme can realize fast mutual authentication, aggregate authentication and session key agreement while achieve high efficiency with high safety. It is more suitable for authentication in large-scale space networks with limited resource.

In future work, how to realize privacy protection of the node will be the key issue in the next step. The space network is a high exposure network, it is easy to be attacked, so we should take steps to achieve anonymous aggregate authentication in the space network.

\section{Acknowledgements}

This paper is supported by the National Key Research and Development Program of China (No.2016YFB0501900), the National Programs for High Technology Research and Development of China (No. 2015AA016006) and the National Natural Science Foundation of China (No. 61502531).

\section{References}

[1] Kambourakis G, Rouskas A, Kormentzas G, et al. Advanced SSL/TLS-based authentication for secure WLAN-3G interworking. IEE Proceedings - Communications. 2004, 151 (5): 501-506.

[2] Yang G, Wong D S, Deng X. Anonymous and Authenticated Key Exchange for Roaming Networks. IEEE Transactions on Wireless Communications. 2007, 6 (9): 3461-3472.

[3] Hou H F, Liu G Q, Ji X S, et al. Provable security authentication scheme based on public key for heterogeneous wireless network. Journal of Electronics \& Information Technology. 2009, 31 (10): 2385-2391(in Chinese).

[4] He D, Ma M, Zhang Y, et al. A strong user authentication scheme with smart cards for wireless communications. Computer Communications. 2011, 34 (3): 367-374.

[5] Mahshid M K, Eslamipoor R. An optimized authentication protocol for mobile networks. Neural Computing and Applications. 2014, 25 (2): 1-7.

[6] Mallissery S, Pai M M M, Smitha A, et al. Improvising the Public Key Infrastructure to Build Trust Architecture 
for VANET by using Short - Time Certificate Management and Merkle Signature Scheme. Ultrasound in Obstetrics \& Gynecology. 2014, 44 (S1): 40-40.

[7] A. Shamir, Identity based cryptosystems and signature schemes. In: G.R.Blakley, D. Chaum (Eds.). Crypto'84, LNCS 196, Springer-Verlag, Santa Barbara,California, USA, 1984, pp. 47-53.

[8] Kate A, Zaverucha G M, Hengartner U. Anonymity and security in delay tolerant networks. International Conference on Security and Privacy in Communications Networks and the Workshops. 2007. SECURECOMM. IEEE Xplore, 2007, pp. 504-513.

[9] Yang G, Huang Q, Wong D S, et al. Universal authentication protocols for anonymous wireless communications. IEEE Transactions on Wireless Communications. 2010, 9 (1): 168-174.

[10] Jiang Q, Ma J F, Li G S, et al. Identity-based roaming protocol with anonymity for heterogeneous wireless networks. Journal on Communications. 2010, 31 (10): 138-145(in Chinese).

[11] Lo N W, Tsai J L. An Efficient Conditional Privacy-Preserving Authentication Scheme for Vehicular Sensor Networks Without Pairings. IEEE Transactions on Intelligent Transportation Systems. 2016, 17 (5): 1319-1328.

[12] S. Al-Riyami, K. Paterson, Certificateless public key cryptography. In: C.S. Laih(Ed.). ASIACRYPT 2003, LNCS 2894, Springer-Verlag, Taipei, 2003, pp. 452-473.

[13] D. Boneh, C. Gentry, B. Lynn, H. Shacham, Aggregate and verifiably encrypted signatures from bilinear maps. In: E. Biham (Ed.). EUROCRYPT 2003, LNCS2656, Springer-Verlag, Warsaw, Poland, 2003, pp. 416-432.

[14] Gong Z, Long Y, Hong X, et al. Two Certificateless Aggregate Signatures From Bilinear Maps. Eighth Acis International Conference on Software Engineering, Artificial Intelligence, Networking, and Parallel/distributed Computing. IEEE Computer Society, 2007, pp. 188-193.

[15] Zhang L, Zhang F. A new certificateless aggregate signature scheme. Computer Communications. 2009 , 32 (6): $1079-1085$.

[16] Zhang L, Qin B, Wu Q, et al. Efficient many-to-one authentication with certificateless aggregate signatures. Computer Networks. 2010, 54 (14): 2482-2491.

[17] Liu H, Shi R H, Zhang S, et al. Efficient anonymous roaming authentication scheme using certificateless aggregate signature in wireless network. Journal on Communications. 2016, 37 (7): 182-192 (in Chinese).

[18] Xiong H, Guan Z, Chen Z, et al. An efficient certificateless aggregate signature with constant pairing computations. Information Sciences. 2013, 219 (10): 225-235.

[19] Chen H, Wei SM, Zhu CJ, Yang Y. Secure certificateless aggregate signature scheme. Journal of Software. 2015, 26 (5): 1173-1180 (in Chinese).

[20] Liu H, Wang S, Liang M, et al. New Construction of Efficient Certificateless Aggregate Signatures. International Journal of Security \& Its Applications. 2014, 8 (1): 411-422.

[21] Sun H M, He B Z, Chen C M, et al. A provable authenticated group key agreement protocol for mobile environment. Information Sciences. 2015, 321 (10): 224-237.

[22] Xu G Y, Chen X Y, Du X H, An authentication scheme using hierarchical identity based signature in large-scale delay tolerant networks. Journal of Electronics \& Information Technology. 2013, 35 (11): 2615-2622(in Chinese). 